\title{
Calidad de vida en los niños que viven con enfermedad renal crónica*
}

\author{
Quality of Life in Children Living with Chronic Kidney Disease \\ Qualidade de vida em crianças vivendo com doença renal crônica
}

\author{
Herly Ruth Alvarado a \\ Pontificia Universidad Javeriana, Colombia \\ herly.alvarado@javeriana.edu.co \\ ORCID: http://orcid.org/0000-0001-6316-0122 \\ Alexander Casallas Vega \\ Universidad El Bosque, Colombia \\ ORCID: http://orcid.org/0000-0002-3289-7241 \\ David Andrade Fonseca \\ B. Braun Avitum, Colombia \\ ORCID: http://orcid.org/0000-0002-7233-9041 \\ Ana María Bertolotto \\ Pontificia Universidad Javeriana, Colombia \\ ORCID: http://orcid.org/0000-0001-9795-6866
}

DOI: https://doi.org/10.11144/Javeriana.ie21-2.cvnv

Fecha de recepción: 21 Marzo 2019

Fecha de aprobación: 09 Agosto 2019

Fecha de publicación: 30 Noviembre 2019

\section{Resumen:}

Introducción: la enfermedad renal en los niños causa diferentes cambios que pueden alterar su calidad de vida; por esta razón, es importante que el profesional de salud busque estrategias que contribuyan a disminuir las secuelas y mejorar la calidad de vida del niño. Objetivo: caracterizar la calidad de vida de los niños de 8 a 18 años, con diagnóstico de enfermedad renal crónica, según el instrumento KIDSCREEN-27 de una institución de salud de la ciudad de Bogotá (Colombia). Método: estudio descriptivo de corte transversal con una población de estudio de 62 niños con diagnóstico de enfermedad renal crónica con edades entre 8 y 18 años que asistieron a la consulta de nefrología pediátrica. Resultados: se analizaron cinco dimensiones, donde cada una tuvo un puntaje ponderado mayor a 70 , que se relaciona con una buena calidad de vida; la dimensión con menor puntaje, la de actividad física, tuvo un puntaje de 70,1, y la dimensión de estado de ánimo y sentimientos obtuvo el mayor puntaje, 80,8. Conclusiones: la enfermedad renal crónica puede alterar la calidad de vida, sobre todo en la dimensión de actividad física y entorno escolar. Es primordial que se planteen programas que tengan en cuenta las cinco dimensiones, especialmente las que obtuvieron menor puntaje, con el fin de mejorar la calidad de vida de los niños con esta enfermedad.

Palabras clave: niños, calidad de vida, enfermedad renal crónica.

\section{Abstract:}

Introduction: In children the kidney disease causes different changes that may affect their quality of life. Therefore, it is important for a health professional to find a strategy contributing to reduce the sequelae and improve their quality of life. Objective: To characterize the quality of life in children with ages of 8-18 years who were diagnosed with chronic kidney disease, based on the instrument KIDSCREEN-27 prepared by health center in Bogotá (Colombia). Method: This is a cross-sectional descriptive study with a study population of 62 children diagnosed with chronic kidney disease, with ages from 8 to 18 years, who attended to pediatric nephrology treatment. Results: Five dimensions were analyzed, each with a weighted score above 70 , which indicates a good quality of life. The dimensions with the lowest score [70.1] is the physical activity. The highest score [80.8] was found in the dimension of mood and feelings. Conclusions: The chronic kidney disease may alter the quality of life, mostly regarding the dimension of physical activity and the school environment. It is crucial to set out programs that consider the five dimensions, especially those with the lowest scores, in order to improve the quality of life among the ill children.

Keywords: children, quality of life, chronic kidney disease.

Notas de autor

\footnotetext{
${ }^{a}$ Autora de correspondencia. Correo electrónico: herly.alvarado@javeriana.edu.co
} 


\section{Resumo:}

Introdução: A doença renal nas crianças causa diferentes alterações que podem mudar sua qualidade de vida; por tal razão, é importante que o profissional de saúde procure estratégias que contribuam para reduzir sequelas e melhorar a qualidade de vida da criança. Objetivo: Caracterizar a qualidade de vida das crianças de 8 a 18 anos com diagnóstico de doença renal crônica, segundo o instrumento KIDSCREEN-27 de uma instituição de saúde da cidade de Bogotá (Colômbia). Método: Estudo descritivo transversal com população de estudo de 62 crianças com diagnóstico de doença renal crônica, entre 8 e 18 anos de idade, que atendidas no ambulatório de nefrologia pediátrica. Resultados: Foram analisadas cinco dimensões, nas quais cada uma obteve pontuação ponderada maior que 70, relacionada à boa qualidade de vida; a dimensão com menor pontuação, a de atividade física, obteve pontuação de 70,1, e a dimensão de humor e sentimentos obteve a maior pontuação, 80,8. Conclusões: A doença renal crônica pode alterar a qualidade de vida, especialmente na dimensão de atividade física e entorno escolar. É essencial projetar programas que levem em conta as cinco dimensões, especialmente as que obtiveram pontuações mais baixas a fim de melhorar a qualidade de vida das crianças com esta doença.

Palavras-chave: crianças, qualidade de vida, doença renal crônica.

\section{Introducción}

La enfermedad renal crónica (ERC) se considera el daño renal irreversible que persiste durante tres meses o más, por daños estructurales o funcionales del riñón que afectan o no la tasa de filtración glomerular y ocasionan alteraciones sanguíneas y en la composición de la orina (1). Estos pacientes requieren múltiples y frecuentes estudios, dentro de los que se encuentran hemogramas, química sanguínea, pruebas de función renal, imágenes diagnósticas y biopsia renal. Esta enfermedad produce complicaciones específicas a corto, mediano y largo plazo, como problemas en el desarrollo y crecimiento, al igual que enfermedades metabólicas, óseas, nutricionales, psicosexuales y cardiovasculares (2,3), que generan afectaciones sistémicas. Es menos frecuente en los niños y produce cambios en la vida escolar, en la relación con sus pares e incluso en sus propios intereses $(4,5)$, producto de los frecuentes controles médicos, tratamientos farmacológicos e ingresos hospitalarios (6).

En cuanto a los datos epidemiológicos, se considera que existe un subregistro de la información en relación con la población pediátrica con ERC, debido a que en sus primeros estadios no manifiesta síntomas y signos (7). Por esta razón, se sugiere que se investigue un poco más en este grupo poblacional.

Actualmente, los datos señalan un estimado de entre 10 y 12 pacientes por millón (PPM) y prevalencias alrededor de 59 a 74 niños por millón, y más frecuente en niños que en niñas. En los primeros años, la enfermedad se asocia más con alteraciones estructurales de las vías urinarias, como: reflujo vesicoureteral, hidronefrosis, valvas de la uretra posterior, glomerulonefritis aguda, síndrome nefrótico e hipoplasia renal y glomerulopatías $(7,8,9)$. En niños y niñas, entre 5 y 14 años de edad, la enfermedad renal la causan enfermedades hereditarias, sistémicas o síndrome nefrótico (10).

El informe de enfermedad renal crónica de Saran et al. (11) menciona que para 2017 Taiwán era el país con la mayor incidencia y prevalencia de ERC, con una cifra de 476 PPM de habitantes y 3317, respectivamente. En Suramérica, Chile es el país con la mayor incidencia (con 180 PPM). En Nefrología básica 2, de la Asociación Colombiana de Nefrología (12), se señaló que la incidencia para Colombia fue 112 PPM y que la prevalencia fue de 624 PPM; sin embargo, es preciso recordar, como se mencionó, que los datos no se conocen con exactitud (12).

En el reporte de enfermedades de alto costo de 2017, para Colombia se notificaron 278 casos reportados en niños de 0 a 4 años; 405, de 5 a 9 años; 725, de 10 a 14 años, y entre 15 y 19 años se notificaron 1725, para un total de 3120 casos de niños con ERC (13).

La ERC era responsable de gran número de muertes tanto en niños como en adultos; pero gracias al avance tecnológico, la sobrevida ha mejorado. Sin embargo, la calidad de vida tanto del niño que la sufre como la de su familia se puede ver afectada por las complicaciones que provoca $(14,15)$. 
La Organización Mundial de la Salud define la calidad de vida relacionada con la salud (CVRS) como "las metas, expectativas, estándares o preocupaciones de un niño sobre su salud en general y los dominios relacionados con la salud" $(16,17)$. Este concepto es multidimensional e incluye la función física, social y emocional del niño y su familia, y se trastoca en la mayoría de los niños con enfermedades crónicas, especialmente en aquellos con nivel socioeconómico bajo $(18,19,20,21)$.

El resultado de la medición de la CVRS es considerado un importante indicador de resultados en la evaluación de las intervenciones y tratamientos y resulta útil para identificar y priorizar los problemas de salud que se presentan en la población pediátrica con ERC y plantear programas que permitan disminuir las disparidades de cuidado con los niños que no presentan esta enfermedad $(22,23)$.

En este aspecto se han descrito diferentes instrumentos para evaluar la calidad de vida en los niños; no obstante, estos se han desarrollado para otro tipo de población y contextos diferentes; por esta razón, se decidió utilizar una medida genérica, denominada KIDSCREEN-27, que mide la CVRS en niños y adolescentes con enfermedades crónicas al abordar cinco dimensiones: actividad física, estado de ánimo y sentimientos, vida familiar y tiempo libre, apoyo social y amigos y entorno escolar (24).

Es importante que el profesional de enfermería tenga en cuenta que la enfermedad se puede controlar y prevenir si se realizan buenos controles y un buen examen físico. También es preciso cuidar de que los síntomas en los niños no pasen inadvertidos, ya que como se dijo pueden estar cursando las etapas iniciales de esta enfermedad.

\section{Objetivo general}

Caracterizar la calidad de vida de los niños de 8 a 18 años con diagnóstico de ERC, según el instrumento KIDSCREEN-27 de una institución de salud de la ciudad de Bogotá (Colombia).

\section{Materiales y métodos}

Se realizó un estudio descriptivo de corte transversal con una población de estudio de 62 niños con diagnóstico de ERC con edades entre los 8 y los 18 años que asistieron a la consulta de nefrología pediátrica. En este estudio se midieron variables de interés con el propósito de determinar la frecuencia de la magnitud y la distribución de variables vinculadas a la calidad de vida de la población estudiada.

Se incluyeron niños que tuvieran diagnóstico establecido de ERC por parte de un nefrólogo pediatra, al menos, dos meses antes de la recolección de datos, con edades de 8 a 18 años, que la lengua nativa fuera español, que aceptaran firmar asentimiento informado y contaran con firma del consentimiento informado por parte de los padres. Por otro lado, se excluyeron aquellos niños que presentaran trastornos psiquiátricos diagnosticados y consignados en la historia clínica, como trastorno depresivo mayor, intento suicida y abuso de sustancias psicoactivas; también aquellos que tuvieran incapacidad física o cognitiva que les impidiera entender o contestar el instrumento, aquellos que hubieran presentado un episodio agudo que necesitaran hospitalización durante las últimas ocho semanas y, finalmente, los niños en condición de analfabetismo. El instrumento era autodiligenciado por el niño; sin embargo, siempre contaban con acompañamiento por la persona encargada de recolectar la muestra por si presentaba dudas sobre alguna de las preguntas.

La investigación fue aprobada por el Comité de Ética e Investigación de la Facultad de Enfermería de la Pontificia Universidad Javeriana y Hospital Universitario San Ignacio. Este estudio conservó los lineamientos establecidos en la Resolución 008430 de 1993, del Ministerio de Salud de Colombia (25). Se contempló el consentimiento informado, la participación voluntaria y el manejo confidencial de la información.

El instrumento para recolectar los datos fue el KIDSCREEN-27, que se desarrolló como una versión reducida del KIDSCREEN-52. Este instrumento ha sido validado para población colombiana y para este 
estudio se adquirieron las licencias de utilización (26,27,28). El cuestionario consta de 27 ítems y tiene un alfa de Cronbach para cada una de las dimensiones superior a 0,87. Para actividad física es 0,93, para estado de ánimo es 0,96, para vida familiar es 0,92 , para apoyo social y amigos es 0,98 y para entorno escolar es 0,85 (29). Luego de la recolección de datos se controló la calidad de la información, se evitó la presencia de datos perdidos y se evaluó la plausibilidad de cada dato, que lo ameritó de acuerdo con el criterio de los expertos clínicos. En cuanto al análisis estadístico para las variables cuantitativas, se realizaron medidas de tendencia central, medidas de dispersión y posición. En el caso de las variables cualitativas, se realizaron tablas de frecuencias y cálculos de distribución porcentual.

Posteriormente, se realizó un análisis bivariado de acuerdo con las variables de interés definidas por la literatura y experticia de los investigadores. Toda la información se trató con el software estadístico R Project (30).

\section{Resultados}

La población de estudio fue de 62 niños que cumplieron con los criterios de inclusión y diligenciaron el instrumento KIDSCREEN versión 27 para Colombia. Dentro de las variables sociodemográficas se encontró que la edad promedio fue de 12,7 años, con una desviación estándar (DE) $\pm 2,8$ años y un percentil 50 de 13 años, lo que evidencia poca dispersión de los datos en cuanto a esta variable.

En cuanto al género, predominó levemente el masculino, con una proporción del 53,2\#. Los niños principalmente pertenecían a estratos socioeconómicos del 1 al 3, representados en una proporción del 82,3 $\%$ (tabla 1).

TABLA 1.

Distribución de frecuencia absoluta y porcentual de estrato socioeconómico de los niños con enfermedad renal crónica

\begin{tabular}{lrrr}
\hline Estrato socioeconómico & $\mathbf{n}$ & $\%$ & \% acumulado \\
\hline Uno & 13 & 21 & 21 \\
\hline Dos & 28 & 45,2 & 66,2 \\
\hline Tres & 10 & 16,1 & 82,3 \\
\hline Cuatro & 4 & 6,5 & 88,8 \\
\hline Cinco & 1 & 1,6 & 90,4 \\
\hline Seis & 1 & 1,6 & 92 \\
\hline No sabe/no responde & 5 & 8 & 100 \\
\hline Total & 62 & 100 & 100 \\
\hline
\end{tabular}

Fuente: elaboración propia

En cuanto a las características clínicas de los participantes, se tuvieron en cuenta variables como realización de terapia de reemplazo renal, si toma algún medicamento de forma crónica y si había estado hospitalizado durante el último año previo a la aplicación del KIDSCREEN-27 (tabla 2).

TABLA 2.

Características del tratamiento recibido por los niños con enfermedad renal crónica

\begin{tabular}{lrrrrrr}
\hline & \multicolumn{2}{c}{ Diálisis } & \multicolumn{2}{c}{$\begin{array}{c}\text { Consumo de } \\
\text { medicamentos }\end{array}$} & \multicolumn{2}{c}{$\begin{array}{c}\text { Hospitalizaciones } \\
\text { durante el último año }\end{array}$} \\
\hline Respuesta & $\mathbf{n}$ & $\%$ & $\mathbf{n}$ & $\%$ & $\mathrm{n}$ & $\%$ \\
\hline Sí & 1 & 1,6 & 24 & 38,7 & 11 & 17,7 \\
\hline No & 61 & 98,4 & 38 & 61,3 & 51 & 82,3 \\
\hline Total & 62 & 100 & 62 & 100 & 62 & 100 \\
\hline
\end{tabular}

Fuente: elaboración propia 
Del estudio, 11 niños estuvieron hospitalizados, 9 estuvieron hospitalizados una vez durante el último año y 2 refirieron que habían estado hospitalizados 3 veces. De los 24 niños que consumían medicamentos, 15 tomaban entre 1 y 3 medicamentos y los 9 restantes tomaban entre 4 y 5 medicamentos. Los más consumidos fueron antihipertensivos, con el 25\% (figura 1). En otros medicamentos, se agruparon varios tipos de fármacos que la frecuencia absoluta entre los niños no superó 1.

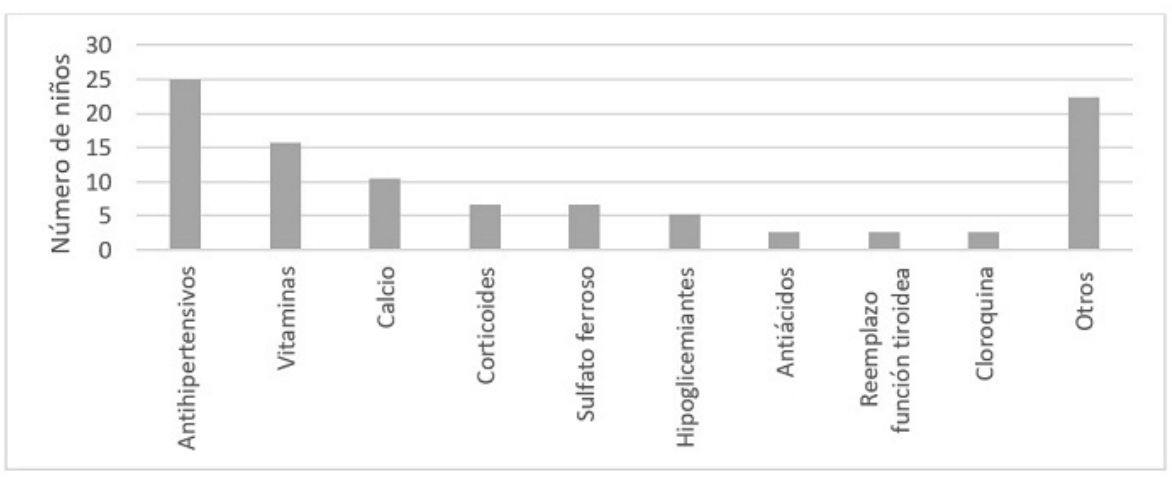

FIGURA 1.

Tipo de medicamentos usados por los niños con enfermedad renal crónica Fuente: elaboración propia

Se indagó si los niños participantes percibieron algún tipo de limitación física, de los cuales solo 7 participantes refirieron tenerla; 4 indicaron tener limitación en la visión, 2 con limitación en el desplazamiento y uno con limitación auditiva.

Para la interpretación del Índice de Masa Corporal (IMC) se tuvo en cuenta la tabla del IMC para la edad de entre 5 y 19 años para cada sexo de la Organización Mundial de la Salud. En ambos sexos no se observaron valores atípicos; sin embargo, tanto en el caso de los niños como en el de las niñas se presentaron 3 casos de sobrepeso y uno con obesidad para un total de 8 participantes con alteración del IMC según la edad (figura 2).

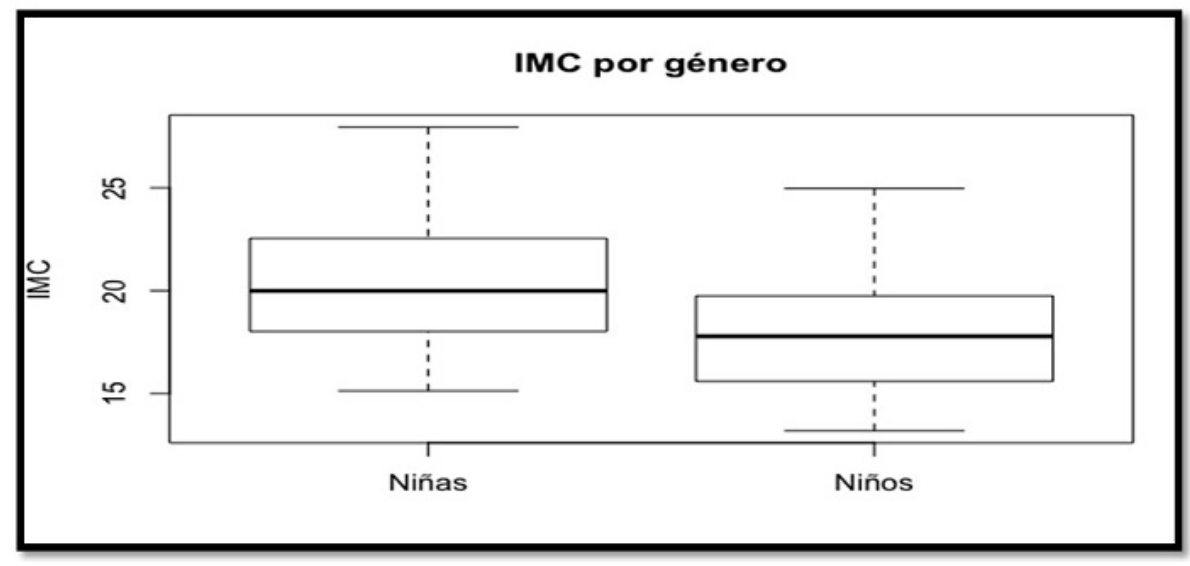

FIGURA 2.

Medidas de posición, tendencia central y dispersión del estado nutricional de los niños con enfermedad renal crónica, según sexo Fuente: elaboración propia

En la evaluación del KIDSCREEN versión 27 para Colombia se analizaron 5 dimensiones contempladas en la escala: 1) actividad física, 2) estado de ánimo y sentimientos, 3) vida familiar y tiempo libre, 4) apoyo social y amigos y 5) entorno social. Acorde con las indicaciones del manual de KIDSCREEN-27, la ponderación de los resultados para calidad de vida debe analizarse de forma particular y no en un acumulado general (tabla 3). Se destacó dentro de esta población de niños que en todas las dimensiones se encontraron 
puntajes por encima de 70, y dentro de estas la actividad física fue la que menos puntaje tuvo, con un 70,1\#; por el contrario, la dimensión "estado de ánimo y sentimientos" fue la del promedio más alto. Pese a que hubo poca dispersión, es importante resaltar que se hallaron niños que en las diferentes dimensiones su ponderado individual no supero el 30, como fue el caso de la dimensión "apoyo social y amigos", en la cual se observó un puntaje de 19. Por otra parte, en todas las dimensiones se logró el puntaje máximo de 100 en cada una de las dimensiones.

TABLA 3.

Calidad de vida por dimensiones del KIDSCREEN-27, Colombia

\begin{tabular}{lrrrrr}
\hline Dimensión & Media & $\begin{array}{r}\text { Desviación } \\
\text { estándar }\end{array}$ & Mediana & Mín.-máx. & RIQ \\
\hline Actividad física & 70,1 & $\pm 19,1$ & 70 & $30-100$ & 27,5 \\
\hline $\begin{array}{l}\text { Estado de ánimo y } \\
\text { sentimientos }\end{array}$ & 80,8 & $\pm 15,2$ & 81,7 & $33-100$ & 20,8 \\
\hline $\begin{array}{l}\text { Vida familiar y tiempo } \\
\text { libre }\end{array}$ & 77,9 & $\pm 15,2$ & 80 & $47-100$ & 27,5 \\
\hline Apoyo social y amigos & 77,9 & $\pm 20,9$ & 84,4 & $19-100$ & 26,6 \\
\hline Entorno escolar & 78,6 & $\pm 18,4$ & 81,3 & $31-100$ & 25 \\
\hline
\end{tabular}

Fuente: elaboración propia

$\mathrm{Al}$ realizar el análisis bivariado entre la dimensión de actividad física y la presencia de hospitalización durante el último año, se encontró que los puntajes obtenidos por los niños que habían estado hospitalizados en el último año fue menor que en los niños que no habían sido hospitalizados, ya que en el primer grupo el resultado fue 60,9 $\pm 24,8$, en comparación con el 72,1 $\pm 17,5$ del segundo grupo. Esto mismo se observó al realizar el análisis bivariado con el consumo de medicamentos y actividad física, donde se encontró que la puntuación de los que consumen medicamentos fue de 66,6 \pm 14,3 y en los que no consumían medicamentos, $72,4 \pm 21,8$ (tabla 4 ).

TABLA 4.

Dimensiones del KIDSCREEN-27 para consumo de medicamentos y previas hospitalizaciones

\begin{tabular}{lrrrr}
\hline \multirow{2}{*}{ Dimensión } & \multicolumn{2}{r}{ Previas hospitalizaciones } & \multicolumn{2}{r}{ Consumo de medicamentos } \\
\cline { 2 - 5 } & $\begin{array}{r}\text { Sí } \\
(\mathbf{n}=11)\end{array}$ & $\begin{array}{r}\text { No } \\
(\mathbf{n}=51)\end{array}$ & $\begin{array}{r}\text { Sí } \\
(\mathbf{n}=25)\end{array}$ & $\begin{array}{r}\text { No } \\
(\mathbf{n}=37)\end{array}$ \\
\hline Actividad fisica & $(60,9) \pm 24,8$ & $(72,1) \pm 17,5$ & $(66,6) \pm 14,3$ & $(72,4) \pm 21,8$ \\
\hline $\begin{array}{l}\text { Estado de ánimo y } \\
\text { sentimientos }\end{array}$ & $(84,8) \pm 12,9$ & $(79,9) \pm 15,7$ & $(81,1) \pm 13,9$ & $(80,5) \pm 16,3$ \\
\hline $\begin{array}{l}\text { Vida familiar y tiempo } \\
\text { libre }\end{array}$ & $(84,2) \pm 10,4$ & $(76,5) \pm 15,9$ & $(75,7) \pm 15,1$ & $(79,4) \pm 15,5$ \\
\hline Apoyo social y amigos & $(73,9) \pm 23$ & $(78,8) \pm 20,7$ & $(82,5) \pm 16,5$ & $(74,8) \pm 23,5$ \\
\hline Entorno escolar & $(79) \pm 22,6$ & $(78,6) \pm 17,8$ & $(78,5) \pm 15,3$ & $(78,7) \pm 20,6$ \\
\hline
\end{tabular}

Fuente: elaboración propia

Una vez se identificó la actividad física como la dimensión con menor ponderación total obtenida para calidad de vida, acorde con los hallazgos de la tabla 4, se procedió con un análisis estadístico de tipo inferencial, en el que se escogió una prueba no paramétrica tipo chi cuadrado. Para el análisis los grupos se dividieron en dos según el manual del usuario de KIDSCREEN-27. El primer grupo de niños fue aquel con un puntaje mayor o igual a 70 (mayor calidad de vida) y el segundo grupo fue el de los niños con menor a 70 (menor calidad de vida). Posteriormente, se determinó si existía alguna asociación con el consumo de medicamentos y el hecho de haber estado hospitalizado.

En el caso de consumo medicamentos, se obtuvo un resultado de $\chi=1,6304, \mathrm{df}=1$; valor de $\mathrm{p}=0,2016 \sin$ una asociación estadísticamente significativa. En el caso de actividad física y hospitalizaciones, se calculó de igual forma la prueba de chi cuadrado con el siguiente resultado: $\chi=4,3131, \mathrm{df}=1$ y valor de $\mathrm{p}=0,0378$. De tal manera que el hecho de tomar medicamentos de forma crónica se asocia estadísticamente con la dimensión de actividad física. 


\section{Discusión}

Los resultados de esta investigación muestran que los niños con ERC se encuentran, en promedio, en edades entre 12,7 años (DE) $\pm 2,8$ años, más alta que la reportada en datos locales (31) y muy cercana a la que se indica en Cuenta de Alto Costo para 2015 (13), lo cual se relaciona con las causas de la enfermedad y la edad en la que inicia. En general, con relación al sexo, la distribución de la enfermedad fue similar, en contravía de estudios donde se menciona que es más frecuente en niños que en niñas, debido a la estructura del aparato urinario (32).

Con respecto al estrato socioeconómico, los datos mostraron que la población de estudio pertenecía en mayor proporción $(82,3 \%)$ a los estratos entre 1 y 3 , datos relacionados con el perfil de paciente que consulta la institución.

Llamó la atención que solo un porcentaje muy bajo de los niños manifestaron estar enfermos y tener algún tipo de limitación física, lo que permite analizar que la mayoría de ellos han introducido la patología a su vida y no la identifican como una condición particular.

Dentro de la evaluación de la calidad de vida con el KIDSCREEN-27 se encontró que la dimensión con menor puntaje y que representa menor calidad de vida fue la "actividad física", aspecto primordialmente afectado por la enfermedad, en cuanto a que lo pacientes deben seguir unas restricciones emitidas por el personal médico y supervisión de los padres, documentado en estudios previos $(20,33)$.

Aspectos como percepción del estado de salud, actividades recreativas, dificultades para correr y nivel de energía percibido se pueden ver afectados por la enfermedad y, en consecuencia, deberían ser actividades orientadas por un programa formal de actividad física, de tal manera que se ajuste a las necesidades del paciente y su condición clínica particular (34).

Las dimensiones de "vida familiar y tiempo libre", "entorno escolar" y "apoyo social y de amigos" fueron las que alcanzaron una medida cerca de 78 puntos; mientras que la mayor puntuación fue documentada en el área de "estado de ánimo y sentimientos", la cual está íntimamente relacionada con aspectos como que los niños sientan que disfrutan la vida, divertirse, sentirse bien con la forma de ser, no sentirse solos o sin ganas de no hacer actividades de la vida diaria.

Con respecto a variables relevantes en el tratamiento y seguimiento de la enfermedad, la mayoría de los niños no eran usuarios de diálisis; sin embargo, más de la mitad recibía de forma rutinaria la administración de medicamentos, y el $24 \%$ de los niños, entre 1 y 5 medicamentos, lo cual resulta ser una medida terapéutica que impacta en cuanto a la calidad de vida, principalmente en las dimensiones de actividad física, vida familiar y tiempo libre, que tiene vínculo directo con la complejidad en la adherencia al tratamiento en el contexto de las enfermedades crónicas (35).

Se encontraron 3 niños con sobrepeso y uno con obesidad, lo cual podría relacionarse con la alteración que ellos perciben frente a la dimensión "actividad física”. También se identificó que la población de estudio presentó un porcentaje apreciable de hospitalizaciones durante el último año a causa de la enfermedad, lo que influye principalmente en las dimensiones "actividad física" y "apoyo social y amigos", debido a que la hospitalización es un entorno en el que hay falta de información, vergüenza debido a la necesidad de permanecer en pijama o bata del hospital y tristeza por la separación de su familia, amigos, colegio y otros (36).

\section{Conclusiones}

La puntuación encontrada con la medida genérica de calidad de vida en los niños con ERC evidenció que la dimensión más afectada fue la de "actividad física", sobre todo en los niños que han requerido hospitalización y que toman medicamentos para el control de la enfermedad. 
Como recomendaciones, se puede proponer que en el programa de atención integral para estos niños se planteen actividades que consideren la condición clínica particular de cada uno de ellos, a fin de mejorar tanto la adherencia a los tratamientos como la CVRS. Es importante también recordar que muy pocos niños son conscientes de tener una enfermedad crónica, y este aspecto es relevante, ya que puede ser muy positivo, al disminuir el efecto negativo en el aspecto psicológico y social; pero puede ser negativo, ya que al no considerarse enfermos, la adherencia a los tratamientos puede ser menor.

Es necesario adaptar transculturalmente una medida específica para medir la CVRS en niños con ERC, y así poder contar con datos mucho más precisos y exactos frente a su calidad de vida.

\section{Limitaciones}

Dentro de las limitaciones de la presente investigación se encuentra que al ser un estudio de tipo descriptivo no se pueden establecer asociaciones o causalidad; sin embargo, permitió establecer hipótesis acerca de realizar más investigaciones específicas sobre la ERC y la movilidad física, a la vez que orientar en la práctica clínica la prioridad de tener en cuenta movilidad y actividad física dentro de las valoraciones y cuidados de los niños con ERC.

\section{Referencias}

1. Zamora I, Sanahuja MJ. Enfermedad renal crónica [Internet]. En Protocolos diagnósticos de la AEP: nefrología pediátrica. Madrid: Asociación Española de Pediatría; 2008 [citado 2019 Jul 4]. p. 231-9. Disponible en: http ://www.aeped.es/protocolos/

2. Hanson CS, Gutman T, Craig JC, Bernays S, Raman G, Zhang Y, et al. Identifying important outcomes for young people with CKD and their caregivers: a nominal group technique study. Am J Kidney Dis. 2019;74(1):82-94.

3. Weaver DJ, Mitsnefes M. Cardiovascular disease in children and adolescents with chronic kidney disease. Semin Nephrol [Internet]. 2018 Nov [citado 2019 Jul 11];38(6):559-69. Disponible en: http://www.ncbi.nlm.nih.go v/pubmed/30413251

4. Kang NR, Ahn YH, Park E, Choi HJ, Kim SH, Cho H, et al. Mental health and psychosocial adjustment in pediatric chronic kidney disease derived from the KNOW-Ped CKD study. Pediatr Nephrol [Internet]. 2019 Jun 20 [citado 2019 Jul 11]. Disponible en: http://www.ncbi.nlm.nih.gov/pubmed/31222663

5. Heath J, Norman P, Christian M, Watson A. Measurement of quality of life and attitudes towards illness in children and young people with chronic kidney disease. Qual Life Res. 2017;26(9):2409-19.

6. Aparicio-López C, Fernández-Escribano A, Garrido-Cantanero G, Luque-de Pablos A, Izquierdo-García E. Influencia de la situación clínica en la calidad de vida relacionada con la salud de los pacientes pediátricos con enfermedad renal crónica. Nefrología [Internet]. 2013 Jan 1 [citado 2019 Feb 20];33(1):61-9. Disponible en: http://www.revistanefrologia.com/es-influencia-situacion-clinica-calidad-vida-relacionada-con-salud-los-p acientes-articulo-X0211699513002668

7. Pérez-Cuevas R, Muñoz-Hernández O. Importancia de la salud pública dirigida a la niñez y la adolescencia en México. Bol Med Hosp Infant Mex [Internet]. 2014 [citado 2018 Sep 12];71(2):126-33. Disponible en: http:/ /www.scielo.org.mx/scielo.php?script=sci_arttext\&pid=S1665-11462014000200010

8. Lopera Medina MM. La enfermedad renal crónica en Colombia: necesidades en salud y respuesta del Sistema General de Seguridad Social en Salud. Rev Gerenc Polít Salud [Internet]. 2016 Jun 30 [citado 2019 Jul 4];15(30):212-33. Disponible en: http://revistas.javeriana.edu.co/index.php/gerepolsal/article/view/17165

9. Rampersad C, Dart A. Widening the lens to childhood. Curr Opin Nephrol Hypertens [Internet]. 2019 May [citado 2019 Jul 11];28(3):233-7. Disponible en: http://www.ncbi.nlm.nih.gov/pubmed/30844883 
10. Ministerio de Salud de Colombia. Enfermedad renal y niños: actúa pronto para prevenirlo [Internet]. 201610 de marzo [citado 2019 Jul 4]. Disponible en: https://www.minsalud.gov.co/Paginas/-Enfermedad-renal-y-niños-a ctua-pronto-para-prevenirlo.aspx

11. Saran R, Robinson B, Abbott KC, Agodoa LYC, Bhave N, Bragg-Gresham J, et al. US Renal Data System 2017 Annual Data Report: Epidemiology of Kidney Disease in the United States. Am J Kidney Dis [Internet]. 2018 Mar 1 [citado 2019 Jul 12];71(3S1):A7. Disponible en: http://www.ncbi.nlm.nih.gov/pubmed/29477157

12. Carlos M, Henao V, Augusto C, Valencia R. Enfermedad renal crónica. En Nefrología básica 2 [Internet]. Bogotá: Asociación Colombiana de Nefrología; 2018 [citado 2019 Jul 4]. Disponible en: http://asocolnef.com/wp-con tent/uploads/2018/06/Capítulo-Enfermedad-Renal-Cronica.pdf

13. Fondo Colombiano de Enfermedades de Alto costo. Situación de la enfermedad renal crónica, hipertensión arterial y la diabetes mellitus en Colombia 2017 [Internet]. Bogotá: Fondo; 2018 [citado 2019 Jul 4]. Disponible en: htt ps://cuentadealtocosto.org/site/images/Publicaciones/2018/Libro_Situacion_ERC_en_Colombia_2017.pdf

14. Webster AC, Nagler E V, Morton RL, Masson P. Chronic Kidney Disease. The Lancet. 2017;389(10075):1238-1252.

15. Didsbury M, van Zwieten A, Chen K, James LJ, Francis A, Kim S, et al. The association between socioeconomic disadvantage and parent-rated health in children and adolescents with chronic kidney disease-the Kids with CKD (KCAD) study. Pediatr Nephrol. 2019;34(7):1237-45.

16. Fayed N, De Camargo OK, Kerr E, Rosenbaum P, Dubey A, Bostan C, et al. Generic patient-reported outcomes in child health research: A review of conceptual content using World Health Organization definitions. Dev Med Child Neurol. 2012;54(12):1085-95.

17. Haverman L, Limperg PF, Young NL, Grootenhuis MA, Klaassen RJ. Paediatric health-related quality of life: what is it and why should we measure it? Arch Dis Child. 2017;102(5):393-400.

18. Jalali-Farahani S, Shojaei FA, Parvin P, Amiri P. Comparison of health-related quality of life (HRQoL) among healthy, obese and chronically ill Iranian children. BMC Public Health. 2018;18(1):1337.

19. Cantrell MA, Kelly MM. Health-related quality of life for chronically ill children. MCN Am J Matern Nurs. 2015;40(1):24-31.

20. Aparicio López C, Fernández Escribano A, Garrido Cantanero G, Luque de Pablos A, Izquierdo García E. Calidad de vida percibida por ninos con enfermedad renal crónica y por sus padres. Nefrología. 2010;30(1):103-9.

21. Didsbury MS, Kim S, Medway MM, Tong A, McTaggart SJ, Walker AM, et al. Socio-economic status and quality of life in children with chronic disease: A systematic review. J Paediatr Child Health. 2016;52(12):1062-9.

22. El Shafei AM, Soliman Hegazy I, Fadel FI, Nagy EM. Assessment of quality of life among children with end-stage renal disease: a cross-sectional study. J Environ Public Health [Internet]. 2018 [citado 2019 Feb 12];2018:8565498. Disponible en: http://www.ncbi.nlm.nih.gov/pubmed/30305825

23. Welch TR. Disparities in care for children with chronic kidney disease. J Pediatr [Internet]. 2018 Nov 1 [citado 2019 Jul 11];202:8-9. Disponible en: http://www.ncbi.nlm.nih.gov/pubmed/30072139

24. Luarte-Martínez S, Rodríguez-Núñez I, Astudillo P, Manterola C. Propiedades psicométricas de las escalas de gravedad de obstrucción bronquial en pediatría: revisión sistemática y metaanálisis. Arch Argent Pediatr. 2017;115(03):241-8. https://doi.org/10.5546/aap.2017.eng.241

25. Resolución 8430 de 1993, por la cual se establecen las normas científicas, técnicas y administrativas para la investigación en salud [internet]. Ministerio de Salud. Disponible en: https://www.minsalud.gov.co/sites/rid/ Lists/BibliotecaDigital/RIDE/DE/DIJ/RESOLUCION-8430-DE-1993.PDF

26. Robitail S, Ravens-Sieberer U, Simeoni M-C, Rajmil L, Bruil J, Power M, et al. Testing the structural and crosscultural validity of the KIDSCREEN-27 quality of life questionnaire. Qual Life Res [Internet]. 2007 Sep 18 [citado 2019 Mar 18];16(8):1335-45. Disponible en: http://www.ncbi.nlm.nih.gov/pubmed/17668291

27. Pardo-Guijarro MJ, Woll B, Moya-Martínez P, Martínez-Andrés M, Cortés-Ramírez EE, Martínez-Vizcaíno V. Validity and reliability of the Spanish sign language version of the KIDSCREEN-27 health-related quality of life questionnaire for use in deaf children and adolescents. Gac Sanit [Internet]. 2013 Jul [citado 2019 Mar 18];27(4):318-24. Disponible en: http://www.ncbi.nlm.nih.gov/pubmed/23287100 
28. Molina GT, Montaño ER, González AE, Sepúlveda PR, Hidalgo-Rasmussen C, Martínez NV, et al. [Psychometric properties of the quality of life questionnaire health related KIDSCREEN-27 in Chilean adolescents]. Rev Me\#dica Chile. 2014 Nov;142(11):1415-21.

29. Quintero CA, Lugo LH, García HI, Sánchez A. Validación del cuestionario KIDSCREEN-27 de calidad de vida relacionada con la salud en niños y adolescentes de Medellín, Colombia. Rev Colomb Psiquiat. 2011 [citado 2019 Jul 14];40(3):470-87. Disponible en: http://www.redalyc.org/articulo.oa?id=80622315008

30. R-Project. En: Encyclopedia of social network analysis and mining. 2018.

31. Piedrahíta Echeverry VM, Prada Meza MC, Vanegas Ruiz JJ, Vélez Echeverry C, Serna Higuita LM, Serrano Gayubo AK, et al. Causas de enfermedad renal crónica en niños atendidos en el Servicio de Nefrología Pediátrica del Hospital Universitario San Vicente de Paúl, de Medellín, Colombia, entre 1960 y 2010. Iatreia. 2011;24(4):347-52.

32. Medeiros M, Andrade Veneros GD, Toussaint Martínez de Castro G, Ortiz Vásquez L, Hernández Sánchez AM, Olvera N, et al. Prevalencia de enfermedad renal en niños aparentemente sanos con antecedente familiar de terapia de reemplazo renal. Bol Med Hosp Infant Mex [Internet]. 2015 Jul 1 [citado 2019 Feb 12];72(4):257-61. Disponible en: http://linkinghub.elsevier.com/retrieve/pii/S1665114615001616

33. Cavagnaro F. Riñón y deportes. Rev Chil Pediatr [Internet]. 2006 Apr [citado 2019 Mar 18];77(2):185-8. Disponible en: http://www.scielo.cl/scielo.php?script=sci_arttext\&pid=S0370-41062006000200010\&lng=e $\mathrm{n} \& \mathrm{nrm}=\mathrm{iso \& t} \operatorname{lng}=\mathrm{en}$

34. Uribe LD, Correas Espeche E, Lirio C, Adragna DM, Monteverde M, Briones L, et al. Programa de actividad fisica en niños con insuficiencia renal crónica. Medicina Infantil [Internet]. 2016 [citado 2019 Mar 18];23(1):159-62. Disponible en: http://www.medicinainfantil.org.ar

35. Conthe P, Márquez Contreras E, Aliaga Pérez A, Barragán García B, Fernández de Cano Martín MN, González Jurado M, et al. Adherencia terapéutica en la enfermedad crónica: estado de la situación y perspectiva de futuro. Rev Clín Esp [Internet]. 2014 Aug 1 [citado 2019 Mar 18];214(6):336-44. Disponible en: https://linkinghub .elsevier.com/retrieve/pii/S0014256514001271

36. Molocho Silva EM, Leiva Ramírez L. Vivencias de los niños escolares hospitalizados en el Servicio de Pediatría del Hospital General de Jaén-Cajamarca, 2016. Univ Nac Pedro Ruiz Gall [Internet]. 2018 [citado 2019 Mar 18]. Disponible en: http://repositorio.unprg.edu.pe/handle/UNPRG/2233

\section{Notas}

* Artículo de investigación

1. Financiación: el estudio fue financiado por la Pontificia Universidad Javeriana

2. Conflictos de interés: ninguno de los investigadores presentó conflictos de interés

Licencia Creative Commons CC BY 4.0

Cómo citar este artículo: Alvarado HR, Casallas Vega A, Andrade Fonseca D, Bertolotto AM. Calidad de vida en los niños que viven con enfermedad renal crónica. Investig Enferm Imagen Desarr. 2020;21(2). https://doi.org/10.11144/Javeriana.ie21-2.cvnv 